\title{
WEAK CUTPOINT ORDERING ON HEREDITARILY UNICOHERENT CONTINUA
}

\author{
R. J. KOCH AND I. S. KRULE
}

1. Introduction. We present here two criteria for determining when an hereditarily unicoherent continuum admits the structure of a partially ordered space which is monotone, closed, and has a unique minimal element (definitions below). Our interest in such partial orderings stems from the fact (Hunter [1]) that a one-dimensional compact connected semigroup with zero and unit admits such a partial ordering. Ward [5] has studied a class of partially ordered spaces, called generalized trees, which he characterized as hereditarily unicoherent continua which admit a closed, order-dense partial order with unique minimal element. We improve his characterization by replacing "order dense" by the weaker "monotone," and we give two other characterizations, one of which is completely topological.

2. Definitions. A partial order $\leqq$ on a space $X$ is a relation on $X$ (a subset of $X \times X$ ) which is reflexive, transitive, and antisymmetric. We assume throughout that $X$ is a Hausdorff space, but not necessarily metrizable. The term arc is used to denote a continuum irreducibly connected between two points. For $x \in X$ we set $L(x)$ $=\{y \in X: y \leqq x\}$, and for $A \subset X, L(A)=\bigcup\{L(x): x \in A\}$. We say that $\leqq$ is monotone if $L(x)$ is connected for each $x \in X$ or continuous if $L\left(A^{*}\right) \subset L(A)^{*}$ for each $A \subset X$. It will be convenient to use the following notion of convergence of sets. If $\Gamma$ is a directed set and if $\left\{A_{\gamma}: \gamma \in \Gamma\right\}$ is a family of subsets of $X$, then we define lim sup $A_{\gamma}$ and $\lim \inf A_{\gamma}$ by: $x \in \lim \sup A_{\gamma}\left(x \in \lim \inf A_{\gamma}\right)$ if for each open set $U$ about $x$ there is a cofinal (residual) subset $\Gamma(U) \subset \Gamma$ with $U \cap A_{\gamma} \neq \square$ for each $\gamma \in \Gamma(U)$. We write $\lim A_{\gamma}=A$ or $A_{\gamma} \rightarrow A$ provided $\lim \inf A_{\gamma}$ $=A=\lim \sup A_{\gamma}$. We shall use the following results of Strother [3]:

(1) $\leqq$ is closed (as a subset of $X \times X$ ) if and only if for any net $\left\{x_{\gamma}\right\}$ in $X$ with $x_{\gamma} \rightarrow x$ we have lim sup $L\left(x_{\gamma}\right) \subset L(x)$;

(2) $\leqq$ is continuous if and only if for any net $\left\{x_{\gamma}\right\}$ in $X$ with $x_{\gamma} \rightarrow x$ we have $L(x) \subset \lim \inf L\left(x_{\gamma}\right)$.

(3) $\leqq$ is closed and continuous if and only if for any net $\left\{x_{\gamma}\right\}$ in $X$ with $x_{\gamma} \rightarrow x$ we have $L\left(x_{\gamma}\right) \rightarrow L(x)$.

In what follows $X$ is an hereditarily unicoherent continuum, that is, a compact connected space in which any two points are contained

Presented to the Society, November 29, 1958, under the title Concerning the weak cutpoint ordering; received by the editors November 12, 1959. 
in a unique minimal continuum. We denote by $[x, y]$ the unique continuum irreducible from $x$ to $y$. Now fix $p \in X$, and define $a \leqq_{p} b$ to mean that any continuum $K$ of $X$ which contains $p$ and $b$ also contains $a$. Then $\leqq_{p}$ is termed the weak cutpoint ordering of $X$ with respect to $p$ and is easily seen to be reflexive and transitive. We note that $\leqq_{p}$ need not be antisymmetric (for example, if $X$ is indecomposable). If however $X$ is arcwise connected (or endowed with any of a number of other properties) then $\leqq_{p}$ is antisymmetric.

\section{Characterizations of generalized trees.}

THEOREM. Let $X$ be an hereditarily unicoherent continuum, and let $p \in X$. The following are equivalent:

(1) $\leqq_{p}$ is a monotone, closed partial order on $X$;

(2) there exists a monotone, closed partial order $\leqq$ on $X$ with unique minimal element $p$;

(3) $X$ is arcwise connected, and for any net $\left\{x_{\gamma}\right\}$ in $X$ it is true that $\left[p, x_{\gamma}\right] \rightarrow[p, x]$ if $x_{\gamma} \rightarrow x$.

If further (1), (2) or (3) holds, then $X$ is locally connected at $p$.

Proof. (1) implies (2). Clear.

(2) implies (3). We note that $X$ is arcwise connected; in fact, each element $x$ of $X$ can be joined by a connected chain to $p$ [2, Corollary $1]$. It follows that $[p, x]$ is a chain and an arc. Let $\left\{x_{\gamma}\right\}$ be a net in $X$ such that $x_{\gamma} \rightarrow x$. We first assert that $[p, x] \subset \lim$ inf $\left[p, x_{\gamma}\right]$ $\subset \lim \sup \left[p, x_{\gamma}\right] \subset \lim \sup L\left(x_{\gamma}\right) \subset L(x)$. We shall show only that $[p, x] \subset \lim i n f\left[p, x_{\gamma}\right]$. Suppose $y \in[p, x]-\lim \inf \left[p, x_{\gamma}\right]$. Then there is an open set $V$ about $y$ with $V \cap\left[p, x_{\gamma}\right]=\square$ for a cofinal set of $\gamma$ 's. Let $A=\left\{\gamma: V \cap\left[p, x_{\gamma}\right]=\square\right\}$, let $B=U\left\{\left[p, x_{\gamma}\right]: \gamma \in A\right\}$, and note that $B^{*}$ is a continuum containing $p$ and $x$ and hence $[p, x]$. Therefore $y \in B^{*}$, so that $V \cap\left[p, x_{\gamma}\right] \neq \square \square$ for some $\gamma \in A$, a contradiction; thus, $[p, x] \subset \lim$ inf $\left[p, x_{\gamma}\right]$. We now show that $\lim \sup \left[p, x_{\gamma}\right]$ $C[p, x]$. Suppose $z \in \lim \sup \left[p, x_{\gamma}\right]$; we assert that $z$ compares with each element of $\lim$ inf $\left[p, x_{\gamma}\right]$. For suppose $y \in \lim \inf \left[p, x_{\gamma}\right]$ and $z$ does not compare with $y$; then there is an open set $U$ about $z$ and an open set $V$ about $y$ with no element of $U$ comparing with any element of $V$. But for some $\gamma,\left[p, x_{\gamma}\right] \cap U \neq \square$ and $\left[p, x_{\gamma}\right] \cap V \neq \square$, so that since $\left[p, x_{\gamma}\right]$ is a chain we have a contradiction. Hence $\{z\}$ $\cup \lim \inf \left[p, x_{\gamma}\right]$ is a chain in $L(x)$, and thus $z \in[p, x]$. We have therefore shown that $\left[p, x_{\gamma}\right] \rightarrow[p, x]$, and consequently (3) is established.

(3) implies (1). We first show that $\leqq_{p}$ is monotone. We assert that for $y \in X,[p, y]=L(y)$; for if $z \in[p, y]$ then each continuum containing $p$ and $y$ also contains $z$ (by unicoherence), and hence $z \in L(y)$. Conversely if $w \in L(y)$, then each continuum containing $p$ and $y$ also 
contains $w$, and in particular $[p, y]$ contains $w$. Hence $[p, y]=L(y)$, and $\leqq_{p}$ is monotone. Note that $[p, y]$ is an arc because of the arcwise connectedness and unicoherence. To establish that $\leqq_{p}$ is antisymmetric suppose $x, y \in X$ with $x \leqq_{p} y$ and $y \leqq_{p} x$. Then since $[p, y]$ $=L(y)=L(x)=[p, x]$ and this set is an arc, we conclude that $x=y$. The remainder of the proof is clear from $\$ 2$.

Assuming (1), (2), or (3) holds, the weak cutpoint ordering is seen from $\S 2$ to be continuous as well as closed. To see that $X$ is locally connected at $p$, suppose $U$ is an open set about $p$. With reference to the weak cutpoint ordering it follows from $[4$, vii] that $U\{L(x): L(x) \subset U\}$ is open and contains $p$, whence the local connectedness at $p$ follows.

4. An example. The following space is an arcwise connected hereditarily unicoherent continuum on which there does not exist a monotone, closed partial order with unique minimal element. The failure is due to the lack of proper convergence, or looking at it another way, closed implies continuous ((1) implies (3)) while the converse does not hold.

Let $A$ be the subset of the plane which consists of the unit segment on the $x$-axis, the unit segment erected over the point $(0,0)$, and the vertical segments of length $1 / 2$ erected over the points with coordinates $\left(1 / 2^{n}, 0\right), n$ a positive integer. Let $B$ be the reflection of $A$ through the line $y=1$, and let $X=A \cup B$. It is easy to see that $X$ has the properties listed above.

\section{REFERENCES}

1. R. P. Hunter, On the semigroup structure of continua, Trans. Amer. Math. Soc. vol. 93 (1959) pp. 356-368.

2. R. J. Koch, Arcs in partially ordered spaces, Pacific J. Math. vol. 9 (1959) pp. 723-728.

3. W. L. Strother, Continuity for multi-valued functions, Tulane University dissertation, 1952.

4. A. D. Wallace, Struct ideals, Proc. Amer. Math. Soc. vol. 6 (1955) pp. 634-638.

5. L. E. Ward, Jr., Mobs, trees, and fixed points, Proc. Amer. Math. Soc. vol. 8 (1957) pp. 798-804.

Louisiana State University 